\title{
CORRELAÇÕES ENTRE CARACTERÍSTICAS FÍSICO-QUUIMICAS E SENSORIAIS EM SUCO DE MARACUJÁ-AMARELO (Passiflora edulis VAR. flavicarpa) DURANTE O ARMAZENAMENTO ${ }^{1}$
}

\author{
Delcio SANDI ${ }^{2, *}$, José B. P. CHAVES ${ }^{2}$, Antonio C. G. SOUZA², Marco T. C. SILVA², Jun e F. M. PARREIRAS ${ }^{2}$
}

\section{RESUMO}

Foram estudadas possíveis correlações entre características físicas, químicas e sensoriais em suco de maracujá-amarelo (Passiflora edulis var. flavicarpa) submetido à pasteurização, durante o armazenamento por 120 dias, sob duas temperaturas $\left(25 \pm 5^{\circ} \mathrm{C}\right.$ e $\left.5 \pm 1^{\circ} \mathrm{C}\right)$. As características físicas e químicas relacionadas a cor, parâmetros "L" (luminosidade), "a" (intensidade de vermelho e verde) e "b" (intensidade de amarelo e azul) de Hunter, os açúcares redutores e não-redutores (sacarose, glucose e frutose), os compostos voláteis (butirato de etila, butirato de hexila, caproato de etila e caproato de hexila) e furfural foram correlacionadas com caracteristicas sensoriais (homogeneidade da cor, cor laranja, aroma característico, aroma floral, aroma doce, sabor característico, sabor estranho, sabor oxidado, sabor cozido, gosto doce e gosto amargo) obtidas por Análise Descritiva Quantitativa. As correlações entre os parâmetros de cor e as características sensoriais de homogeneidade da cor e cor laranja foram baixas. Entretanto, o aumento da cor laranja foi proporcional ao aumento do valor "a" e inversamente proporcional aos valores "L" e "b". Correlações importantes foram observadas entre os compostos voláteis e sabor característico e aroma característico, aroma floral e aroma doce; furfural e sabor oxidado, sabor cozido e sabor estranho. Quanto aos açúcares redutores e não-redutores, enquanto observaram-se correlações significativas entre os teores de sacarose e o aroma doce, aroma floral, sabor característico e gosto doce, correlações significativas e positivas foram observadas entre os açúcares redutores e o sabor estranho e gosto amargo. De acordo com nossas observações, o uso de métodos instrumentais pode ser indicado, como a avaliação do escurecimento por meio de parâmetros colorimétricos de cor, sendo o valor "a" de Hunter, o melhor indicado. Palavras-chave: suco de maracujá-amarelo; análises fisicas e químicas; análise sensorial (ADQ); correlações.

\section{SUMMARY}

CORRELATION BETWEEN PHYSICO-CHEMICAL AND SENSORY CHARACTERISTCS OF YELLOW PASSIN FRUIT JUICE OVER STORAGING TIME. Physical-chemistry and sensory quality characteristics correlations were studied in yellow passion fruit juice (Passiflora edulis flavicarpa) submitted to thermal processing treatment, during 120 days of storage under two different temperatures. The color characteristics, Hunter ' $\mathrm{L}$ ', ' $\mathrm{a}$ ' and ' $\mathrm{b}$ ' values, reducing sugars and non reducing sugars (sucrose, glucose and fructose), volatile compounds (ethyl butyrate, hexyl butyrate, ethyl caproate and hexyl caproate) and furfural were correlated with sensory characteristics (juice homogeneity of color, orange color, characteristic aroma, floral aroma, sweet aroma, characteristic flavor, off flavor, oxidized flavor, cooked flavor, sweet taste and bitter taste) by Quantitative Descriptive Analysis. The color parameters and sensory characteristic correlations such as juice homogeneity of color and orange color, and a-value was proportional, instead ofL and b-values. Important correlations were observed between volatile compounds and characteristics flavor and aroma, sweet and floral aroma; furfural and oxidized, cooked and off flavor. The sucrose levels were statistically correlationated with sweet and floral aroma, characteristic flavor and sweet taste, while the reducing sugars were correlated with off flavor and bitter taste. From the results it can be concluded that the instrumental methods can be indicated, such as the browning color where colorimetric parameters like a-value is the best one. Keywords: yellow passion fruit juice; physical-chemistry analysis; sensory analysis (QDA); statistical correlations.

\section{1 - INTRODUÇÃO}

Originário da América Tropical, o maracujá é largamente cultivado e processado em todo o mundo. Peru, Venezuela, África do Sul, Sri Lanka, Austrália, Quênia, Colômbia, Peru, Equador, Venezuela, Costa Rica, entre outros, são exemplos de países produtores, sendo o Brasil, o maior produtor mundial [22].

Esta fruta tem sido bastante consumida devido ao seu aroma e acidez acentuados, principalmente como suco, e também em uma série de produtos como sorvetes, mousses, bebidas alcoólicas, entre outros.

A participação do Brasil na exportação de sucos vem caindo dada a forte concorrência com países como a Colômbia, Peru e Equador [22], razão pela qual, o investimento em tecnologias são justificados, para me-

Recebido para publicação em 21/09/2001. Aceito para publicação em 27/01/2003 (000741).

2. UFV - Departamento de Tecnologia de Alimentos, CEP 365371-000 Viçosa-MG

* A quem a correspondência deve ser enviada. lhorar a produtividade, e os sistemas de processamento da fruta.

Para a melhoria dos sistemas de processamento, o entendimento das reações físicas e químicas que ocorrem durante a transformação da fruta in natura em produtos derivados, e sua relação com as características sensoriais é de extrema importância, possibilitando a avaliação indireta das características sensoriais por meio de análises instrumentais [1].

Neste sentido, estudos acerca das correlações entre características sensoriais, fisicas e químicas em alimentos têm sido efetuados, com o intuito de, principalmente, substituir as avaliações sensoriais, as quais, geralmente necessitam de bastante tempo para a sua execução, por análises físicas e químicas. Além disso, a avaliação das correlações entre estas características, auxilia no entendimento dos fatores que levam um alimento a alterar o seu perfil sensorial, contribuindo para a otimização dos processos de produção $[1,16]$.

O objetivo deste trabalho foi testar correlações entre algumas características fisicas e químicas e sensoriais, 
em suco de maracujá-amarelo (Passiflora edulis var. flavicarpa), pasteurizado e armazenado durante 120 dias, sob duas temperaturas, $25 \pm 5^{\circ} \mathrm{C}$ e $5 \pm 1^{\circ} \mathrm{C}$.

\section{2 - MATERIAL E MÉTODOS}

\section{1 - Materiais}

Frutos adquiridos na região da Zona da Mata de Minas Gerais foram armazenados em câmara fria por 4 dias, a temperatura de $5 \pm 1^{\circ} \mathrm{C}$, sendo retirados e processados até a obtenção do suco, de acordo com o fluxograma da Figura 1.

Em seguida o suco foi processado na planta de processamento de produtos vegetais da Escola Agrotécnica Federal de Rio Pomba (Rio Pomba, Minas Gerais), utilizando-se um despolpador mecânico de três estágios, modelo DMF-04; um concentrador a vácuo, modelo CVS03; e um pasteurizador tubular multitubos com capacidade para $1500 \mathrm{~L} / \mathrm{h}$, modelo PTU-02. Todos os equipamentos foram fornecidos pela empresa Tecnint (Congonhal, Minas Gerais).

\section{2 - Métodos}

\subsection{1 - Processamento}

Os frutos, após terem sido selecionados e lavados, sofreram o corte e despolpamento. Neste, foram utilizadas duas peneiras: a primeira com malha de $0,5 \mathrm{~mm}$ e a segunda de $0,3 \mathrm{~mm}$.

Em seguida o suco sofreu a adição de benzoato de sódio comercial na concentração de 500ppm sob agitação, sendo então desaerado no concentrador a vácuo à pressão de $750 \mathrm{mmHg}$.

\begin{tabular}{|l|}
\hline Recepção da fruta \\
\hline Seleção e lavagem \\
\hline Corte e extração da polpa \\
\hline Despolpamento \\
\hline Deaeração e adição de conservantes \\
\hline Pasteurização \\
\hline Embalagem \\
\hline \\
\hline Armazenam ento \\
\hline
\end{tabular}

FIGURA 1. Fluxograma para elaboração do suco de maracujá.

Imediatamente, o suco foi pasteurizado utilizandose três binômios equivalentes: $75^{\circ} \mathrm{C} / 60 \mathrm{~s}, 80^{\circ} \mathrm{C} / 41 \mathrm{~s} \mathrm{e}$ $85^{\circ} \mathrm{C} / 27 \mathrm{~s}$, sendo embalado imediatamente em garrafas de vidro de $500 \mathrm{~mL}$, as quais foram fechadas com tam- pas de metal, com recravadeira manual, proporcionando assim a embalagem do suco ainda quente (hot-fill). Após a embalagem, as garrafas contendo o suco foram imediatamente resfriadas em um tonel metálico contendo água corrente a temperatura de $\pm 10^{\circ} \mathrm{C}$. O suco embalado foi então armazenado em prateleiras a temperatura ambiente $\left(25 \pm 5^{\circ} \mathrm{C}\right)$ e à temperatura refrigerada, a $5 \pm 1^{\circ} \mathrm{C}$ em câmara fria, por 120 dias.

Os tratamentos térmicos utilizados (binômios tempo $\mathrm{x}$ temperatura) foram estipulados com base no trabalho de TCHANGO-TCHANGO et al. [25], que encontraram a levedura Candida pelliculosa como microrganismo mais resistente em néctar de maracujá, com um valor de $\mathrm{D}_{75^{\circ} \mathrm{C}}=1,0 \mathrm{~min}$. Dessa forma, estabeleceu-se o binômio $75^{\circ} \mathrm{C} / 60$ s como referência e, a partir dele, foram determinados os binômios equivalentes, ou seja, de igual letalidade microbiológica, segundo o modelo de Bigelow.

O experimento foi conduzido segundo o delineamento em parcelas subdivididas, com tratamento térmico nas parcelas realizado em ordem aleatória, em três niveis $\left(85^{\circ} \mathrm{C} / 27 \mathrm{~s}, 80^{\circ} \mathrm{C} / 41 \mathrm{~s}, 75^{\circ} \mathrm{C} / 60 \mathrm{~s}\right)$ e tempo de estocagem nas subparcelas, em cinco niveis $(0,30,60,90$ e 120 dias) mantidos a temperatura ambiente $\left(25 \pm 5^{\circ} \mathrm{C}\right)$, e refrigerada $\left(5 \pm 1^{\circ} \mathrm{C}\right)$. Os ensaios foram conduzidos em três repetições.

\subsection{2 - Metodologia das análises}

a) Açúcares redutores e não-redutores

Glucose, frutose e sacarose foram determinadas por cromatografia líquida de alta eficiência, em um cromatógrafo HP Series 1050, com detector por indice de refração HP 1047A, utilizando-se uma coluna HP-87H, $300 \mathrm{~mm} \times 7,8 \mathrm{~mm}$. As condições de trabalho foram: fluxo da fase móvel de $0,7 \mathrm{~mL} / \mathrm{min}\left(\mathrm{H}_{2} \mathrm{SO}_{4} \mathrm{0}, 005 \mathrm{~N}\right)$ e temperatura da coluna igual a $60^{\circ} \mathrm{C}$.

As amostras foram preparadas diluindo-se o suco integral na proporção de 1:10 com água destilada e centrifugadas duas vezes. Na primeira, $80 \mathrm{~mL}$ da amostra foram centrifugados a $3.833 \mathrm{xg}$, por 10 minutos, sendo retirados $1,5 \mathrm{~mL}$ do sobrenadante e transferidos para "eppendorfs". Na segunda centrifugação, as amostras foram colocadas em uma microcentrífuga a $77.600 \mathrm{x} g$, durante 20 minutos. Preparadas, as amostras foram congeladas e mantidas sob congelamento $\left(-30^{\circ} \mathrm{C}\right)$ até a análise, realizada no tempo final de armazenamento. Descongeladas no momento da análise, foram então filtradas em filtro de seringa HV Millex em polietileno, com membrana durapore de $13 \mathrm{~mm}$ de diâmetro $(0,45 \mu \mathrm{m}$ de poro) marca Milipore, sendo analisadas imediatamente.

A identificação foi realizada verificando-se o tempo de retenção dos compostos e utilizando-se a técnica de co-eluição. A sua quantificação foi realizada pelo método do padrão externo, utilizando-se padrões dos compostos da marca Merck. Para tal, uma solução-estoque de cada açúcar foi preparada, obtendo-se concentrações exatas para cada um: 301,5mM para sacarose, 299,3mM para glucose e 300,1 mM para frutose. Destas soluções, obteve-se uma mistura dos padrões de concentração igual 
a $100,4 \mathrm{mM}$ para a sacarose, $99,8 \mathrm{mM}$ para a glucose e $100,0 \mathrm{mM}$ para a frutose. Da mistura, obtiveram-se três soluções diluídas, de concentração exata (em torno de $25 \mathrm{mM}, 10 \mathrm{mM}$ e $4 \mathrm{mM}$, respectivamente), que foram utilizadas na obtenção das curvas de calibração.

b) Cor

A cor foi determinada em um colorimetro modelo Colorquest II, do sistema de Hunter, com leitura direta dos valores "L" (luminosidade), "a" (intensidade de vermelho e verde) e " $b$ " (intensidade de amarelo e azul). As amostras foram diluídas na proporção de 1:10, com água destilada, realizando-se a análise em seguida.

c) Análise dos compostos voláteis e furfural

Foram determinados os compostos voláteis: caproato de hexila - FEMA:2572; butirato de hexila - FEMA:2568; caproato de etila - FEMA:2439; butirato de etila FEMA:2427 e furfural - FEMA:2489.

As amostras de suco foram preparadas tomando-se o suco integral diluído na proporção de 1:10 com água destilada, centrifugadas a 3.833 x g por 10 minutos, eliminando-se assim a polpa, que reduz a eficiência da extração dos compostos voláteis no método utilizado.

Da amostra centrifugada, $20 \mathrm{~mL}$ foram transferidos para um recipiente de vidro com capacidade para $25 \mathrm{~mL}$, com tampa de silicone. Assim, a amostra estava pronta para sofrer a extração dos compostos voláteis.

Os compostos voláteis foram extraídos das amostras preparadas, pelo método de Microextração em Fase Sólida (MeFS) [27]. Utilizou-se um holder manual para $\mathrm{MeFS}$, e fibras de polidimetilsiloxano com filme de $100 \mu \mathrm{m}$, marca Supelco. O tempo de extração foi de 20 minutos em imersão, sob agitação da amostra, com tempo de desorção no injetor de 5 minutos.

Utilizou-se um cromatógrafo modelo VARIAN 3400, com detector de ionização de chama; coluna capilar CPSIL 88, marca Chrompack ( $50 \mathrm{~m}$ x 0,25mm i.d.). A técnica de injeção utilizada foi splitless, e as condições de análise foram as seguintes: gás de arraste: hélio $(36 \mathrm{~cm} / \mathrm{s})$; temperatura do injetor: $230^{\circ} \mathrm{C}$; temperatura do detector: $270^{\circ} \mathrm{C}$; periodo splitless: $2 \mathrm{~min}$; temperatura da coluna: $50^{\circ} \mathrm{C}$ por 4 minutos até $220^{\circ} \mathrm{C}$, a $4,5^{\circ} \mathrm{C} / \mathrm{min}$, sendo mantida a $220^{\circ} \mathrm{C}$ por 18 minutos.

A identificação foi feita utilizando-se padrões sintéticos dos compostos selecionados (IFF Essências e Fragrâncias, São Paulo) pela técnica de co-eluição e por meio do tempo de retenção de cada padrão. A quantificação foi realizada pela utilização de padrão externo. Para tal, preparou-se uma solução-estoque para cada padrão e, em seguida, procedeu-se à diluição destas soluções, obtendo-se, assim, quatro soluções contendo uma mistura de cada padrão dos compostos a quantificar.

A solução mais concentrada continha $75 \mu 1$ de butirato de etila, $75 \mu 1$ de caproato de etila, $25 \mu 1$ de butirato de hexila, $50 \mu 1$ de caproato de hexila e $25 \mu 1$ de furfural em $50 \mathrm{~mL}$ de água destilada $(1,48 \mathrm{ppm}$ de butirato de etila, $1,50 \mathrm{ppm}$ de caproato de etila, 0,50ppm de butirato de hexila, 0,99ppm de caproato de hexila e 0,48ppm de furfural), já efetuadas as correções dependendo da pureza de cada padrão, 98,5\%, 99,7\%, 99,6\%, 99,2\% e $96,6 \%$, respectivamente.

Desta solução, obtiveram-se mais quatro soluções, diluindo-se duas vezes a concentração em cada solução, de forma que a menos concentrada continha $0,18 \mathrm{ppm}$ de butirato de etila, 0,19ppm de caproato de etila, 0,06ppm de butirato de hexila, 0,09ppm de caproato de hexila e 0,06ppm de furfural.

Das quatro soluções foram obtidas quatro curvas de calibração, com $R^{2}=0,921 ; 0,999 ; 0.986 ; 0,847 ; 0,981$ para os compostos butirato de etila, caproato de etila, butirato de hexila, caproato de hexila e furfural, respectivamente, as quais foram utilizadas para a quantificação dos compostos.

\section{d) Análise sensorial}

Paralelamente às análises químicas e físicas realizadas no tempo zero e durante o armazenamento aos 30, 60,90 e 120 dias, foi feita a análise sensorial pela técnica de Análise Descritiva Quantitativa [24] utilizando-se equipe de dez provadores selecionados e treinados [19].

Com base nos atributos levantados pelo método da lista prévia, os provadores avaliaram as amostras. A avaliação foi realizada utilizando-se o delineamento em blocos casualizados, sendo que cada provador avaliava todas as amostras constituindo assim, dez blocos (provadores) por amostra. Foram servidas aos provadores três amostras em cada sessão de análise, aleatoriamente, num intervalo de pelo menos 1 hora e 30 minutos entre as sessões.

As amostras foram preparadas $(30 \mathrm{~mL}$ de cada em copos de plástico de $125 \mathrm{~mL}$ à temperatura ambiente) e servidas em cada sessão de análise, diluindo o suco na proporção de 1:10 com água, sendo servidas em seguida em bandejas acompanhadas de água para enxágüe da boca entre a avaliação de cada amostra.

Os dados obtidos nas análises físicas, químicas e sensoriais foram submetidos à análises de correlação simples, utilizando-se o programa estatístico SAS, versão 6.12 [20].

\section{3 - RESULTADOS E DISCUSSÃo}

A Tabela 1 apresenta os valores obtidos para as correlações entre as características fisicas e químicas e os atributos sensoriais (ADQ).

\section{1 - Cor e atributos sensoriais}

As correlações entre as dimensões de cor ("L", "a" e "b") e as características sensoriais homogeneidade da cor e cor laranja foram consideradas baixas. Observa-se pela Tabela 1, que apesar da cor laranja não ter apresentado correlação significativa com as dimensões de cor, ela é proporcional ao valor "a" e inversamente proporcional aos valores "L" e "b". Este fato é um indicador de que o aumento da intensidade de cores durante o armazenamento, como o vermelho (valor "a"), diminui a intensidade de amarelo 
(valor "b"), levando ao escurecimento do suco (- $\Delta \mathrm{L})$. De acordo com PRIMO [17], citado por REMACHA, IBARZ \& GINER [18], o escurecimento não-enzimático pode se dar durante as operações de processamento como pasteurização, concentração e desidratação; no entanto, esta reação é mais intensa durante o armazenamento.

Na Figura 2 estão plotadas as tendências observadas, durante o armazenamento, para o valor "a" de Hunter, e as características sensoriais, homogeneidade da cor, cor laranja, sabor oxidado, sabor característico e gosto doce.

Segundo MACFIE \& HEDDERLEY [9], a prática usual na obtenção de correlações simples é calcular a média obtida de uma série de repetições, e a partir dela, obter a correlação por meio de programas de computador, o que é sabido tende a superestimar o verdadeiro valor da cor- relação. Realmente, a análise visual da tendência gerada pelos dados médios em cada tempo de armazenamento (Figura 2), nos leva a esperar uma correlação maior do que aquela obtida pela análise estatística. Entretanto, os coeficientes de correlação obtidos entre o valor "a" de Hunter, a homogeneidade da cor, cor laranja, sabor oxidado, sabor característico e gosto doce, foram $0,45,0,23$, $0,30,037$, e 0,41, respectivamente. Isto ocorre devido à variabilidade nas repetições, verificada pelo alto coeficiente de variação (dados não apresentados), que é negligenciada nos casos em que somente os valores médios são utilizados para o cálculo dos coeficientes de correlação. O valor $r=0,90$ é obtido para o valor "a" e a cor laranja, quando calculado com os valores médios, entretanto, $r=0,23$ foi o valor obtido neste experimento, com os dados individuais.

TABELA 1. Coeficientes de correlação obtidos entre as características sensoriais, fĩsicas e químicas

\begin{tabular}{|c|c|c|c|c|c|c|c|c|c|c|c|}
\hline & HCOR & CLAR & ARCA & ARDO & ARFL & SACA & SAOX & SACO & SAES & GODO & GOAM \\
\hline "L" & 0,04 & $-0,21$ & 0,13 & 0,02 & 0,06 & 0,04 & $-0,23$ & $-0,19$ & $-0,16$ & $-0,08$ & $-0,13$ \\
\hline "a" & $-0,45^{* *}$ & 0,23 & $-0,28$ & $-0,34^{* *}$ & $-0,38^{* *}$ & $-0,37^{* *}$ & 0,30 ** & $0,42 * *$ & $-0,02$ & $-0,41^{* *}$ & 0,15 \\
\hline "b" & 0,05 & $-0,19$ & 0,24 & 0,11 & 0,12 & 0,10 & $-0,30 * *$ & $-0,19$ & $-0,27$ & $-0,18$ & $-0,17$ \\
\hline $\mathrm{BE}$ & $-0,39 * *$ & $0,43^{* *}$ & $0,39 * *$ & $0,52 * *$ & $0,47 * *$ & $0,34^{*}$ & $-0,59 * *$ & 0,05 & $-0,45^{* *}$ & $0,67 * *$ & 0,01 \\
\hline $\mathrm{CE}$ & 0,00 & $-0,09$ & $0,49 * *$ & $0,42 * *$ & $0,50 * *$ & $0,34^{*}$ & $-0,33^{*}$ & $-0,18$ & $-0,54 * *$ & 0,23 & $-0,42 * *$ \\
\hline $\mathrm{BH}$ & $-0,14$ & $-0,07$ & $0,56^{* *}$ & $0,40 * *$ & $0,54 * *$ & $0,68 * *$ & $-0,51 * *$ & $-0,21$ & $-0,56 * *$ & $0,34^{*}$ & $-0,32 *$ \\
\hline $\mathrm{CH}$ & $-0,28$ & 0,20 & $0,46 * *$ & $0,35^{* *}$ & $0,38 * *$ & $0,42 * *$ & $-0,39 * *$ & 0,00 & $-0,37 * *$ & $0,46 * *$ & $-0,11$ \\
\hline $\mathrm{FU}$ & $-0,03$ & $-0,10$ & $-0,31^{*}$ & $-0,22$ & $-0,27$ & $-0,49 * *$ & $0,51 * *$ & $0,43 * *$ & $0,38 * *$ & $-0,24$ & $0,32 *$ \\
\hline SA & $0,34 *$ & $-0,46^{* *}$ & $0,31^{*}$ & $0,41 * *$ & $0,39^{*}$ & $0,34 *$ & $-0,21$ & $-0,14$ & $-0,33 *$ & $0,34^{*}$ & $-0,28$ \\
\hline GL & $-0,15$ & 0,17 & $-0,13$ & $-0,57 * *$ & $-0,21$ & $-0,23$ & 0,10 & 0,21 & $0,36^{*}$ & $-0,23$ & $0,33^{*}$ \\
\hline FR & $-0,16$ & 0,17 & $-0,13$ & $-0,57 * *$ & $-0,23$ & $-0,25$ & 0,10 & 0,19 & $0,37 *$ & $-0,24$ & $0,33^{*}$ \\
\hline
\end{tabular}

$\mathrm{BE}=$ butirato de etila; $\mathrm{BH}=$ butirato de hexila; $\mathrm{CE}=$ caproato de etila; $\mathrm{CH}=$ caproato de hexila; $\mathrm{FU}=$ furfural; $\mathrm{SA}=$ sacarose; $\mathrm{GL}=$ glucose; $\mathrm{FR}=$ frutose.; $\mathrm{HCOR}=$ homogeneidade da cor; $\mathrm{CLAR}=$ cor laranja; $\mathrm{ARCA}=$ aroma característico; $\mathrm{ARDO}=$ aroma doce; $\mathrm{ARFL}=$ aroma floral; $\mathrm{SACA}=\mathrm{sabor}$ caracteristico; $\mathrm{SACO}=$ sabor cozido; $\mathrm{SAES}$ $=$ sabor estranho; GODO = gosto doce; GOAM = gosto amargo. ${ }^{*}$ significativo a $5 \%$ de probabilidade; ${ }^{* *}$ significativo a $0,1 \%$ de probabilidade.

Contudo, a análise da Figura 2 nos permite verificar a tendência de cada caracteristica sensorial durante o armazenamento, e a sua relação com a tendência no aumento do valor "a".

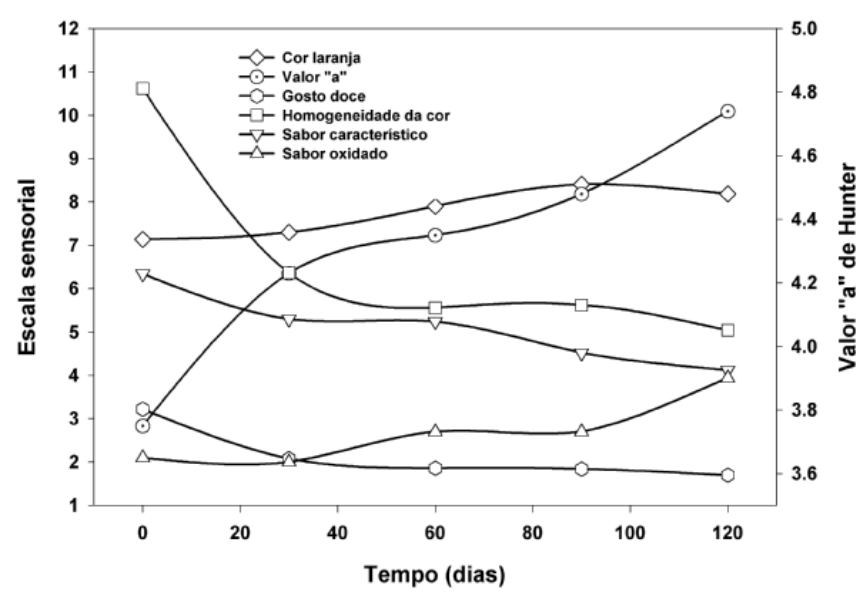

FIGURA 2. Tendência apresentada pelo valor "a" de Hunter, e características sensoriais, obtidas durante o armazenamento.
Mesmo não havendo um coeficiente de correlação alto entre o valor "a" e a cor laranja $(0,23)$, observa-se claramente que o aumento do escurecimento durante o armazenamento, é seguido pelo aumento do valor "a" (Figura 3).

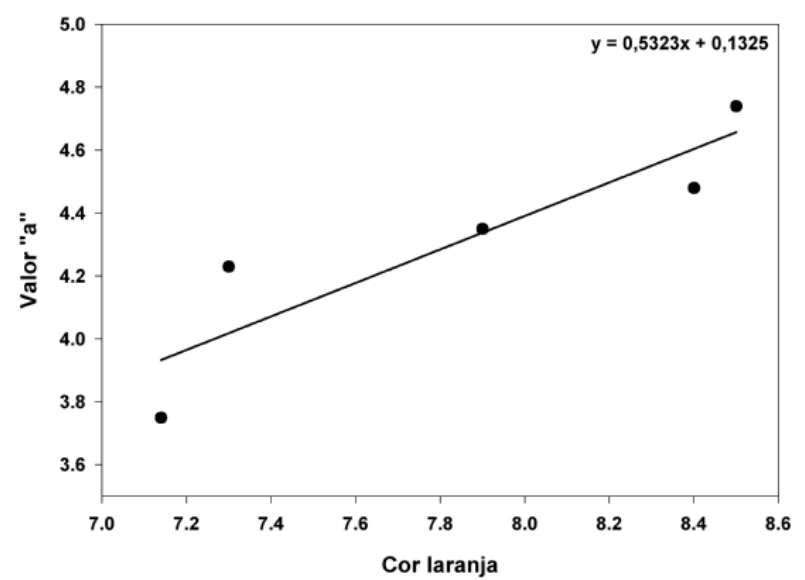

FIGURA 3. Correlação obtida durante o armazenamento entre a cor laranja e o valor "a" de Hunter. 
Pela análise da Tabela 1, observa-se que houve correlação negativa entre os valores de "a" e os atributos considerados desejáveis, por exemplo, o gosto doce $(\mathrm{Fi}$ gura 4) e correlações positivas com atributos indesejáveis, como o sabor cozido. Estes resultados indicam que a diminuição do gosto doce e o aumento do sabor cozido, durante o armazenamento são acompanhados pelo aumento da intensidade de vermelho.

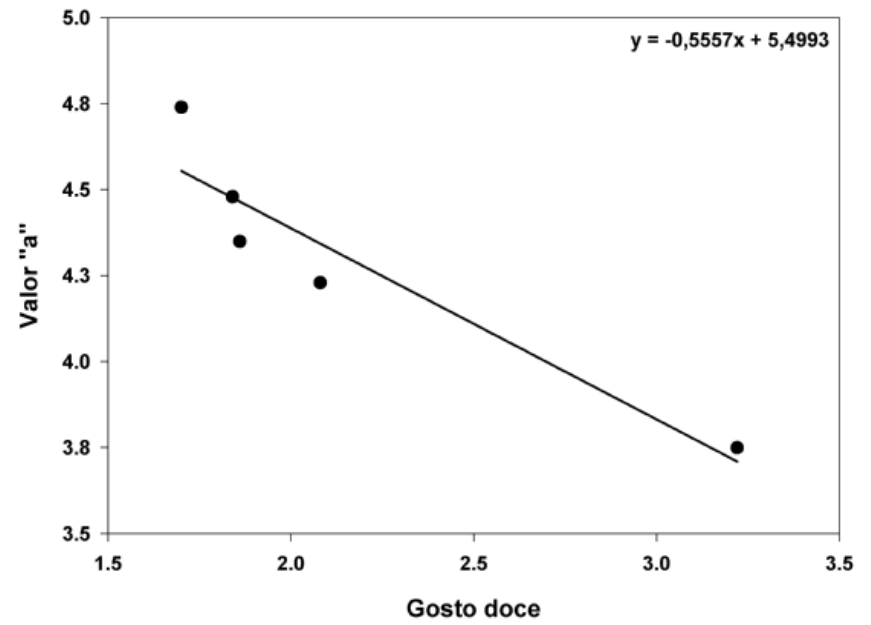

FIGURA 4. Correlação obtida durante o armazenamento entre o gosto doce e o valor "a" de Hunter.

\section{2 - Açúcares e atributos sensoriais}

Os coeficientes de correlação entre a sacarose e o aroma doce $(0,41)$, aroma floral $(0,39)$, sabor característico $(0,34)$ e gosto doce $(0,34)$ foram significativos $(\alpha=5 \%)$, exceto para o aroma floral. Isto demonstra que a medida em que ocorre a diminuição dos teores de sacarose, provocada pela sua inversão, durante o armazenamento, os atributos desejáveis diminuem o seu valor. Por outro lado, correlações significativas foram observadas entre os açúcares redutores e sabor estranho e gosto amargo, demonstrando que à medida que ocorre a inversão da sacarose, aumentando os teores de açúcares redutores, a qualidade sensorial do suco diminui. A Figura 5 demonstra a alta correlação existente entre o aumento dos teores de glucose e frutose $(R=1,00)$, devido à inversão da sacarose. É importante observar que com a inversão da sacarose e um conseqüente aumento no teor de açúcares redutores, uma série de reações nãoenzimáticas ocorre, levando-se ao escurecimento do suco, e a uma série de compostos indesejáveis sensorialmente.

\section{3 - Compostos voláteis e atributos sensoriais}

Em relação aos compostos voláteis, foram observados indices significativos $(\alpha=0,1 \%)$ entre o aroma característico e o butirato de etila $[7,11,14]$, caproato de etila, [6, 11], butirato de hexila, (Figura 6) [7], e caproato de hexila, 0,39, 0,49, 0,56, e 0,46, respectivamente. De acordo com MURRAY, SHIPTON \& WHITFIELD [12], o butirato de etila e o caproato de etila estão entre os três compostos que conferem maior intensidade no aroma de suco de maracujá.

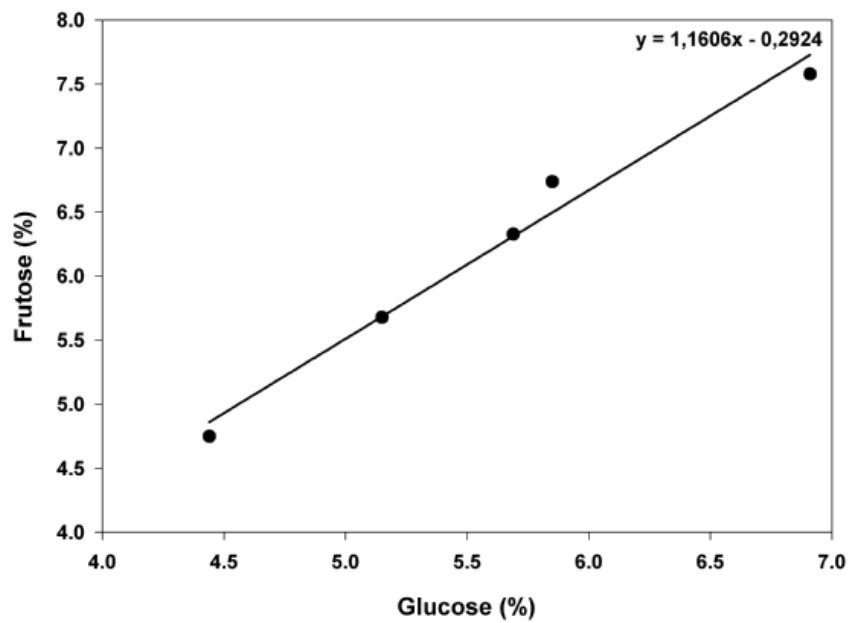

FIGURA 5. Correlação obtida durante o armazenamento, entre os teores de glucose e frutose.

Portanto, a diminuição do aroma característico, detectada sensorialmente, foi acompanhada pela diminuição das concentrações dos compostos voláteis, o que era esperado, uma vez que, com o tempo, diversas reações complexas ocorrem, dentre elas a oxidação, provocando a sua degradação, e a conseqüente diminuição da sua concentração.

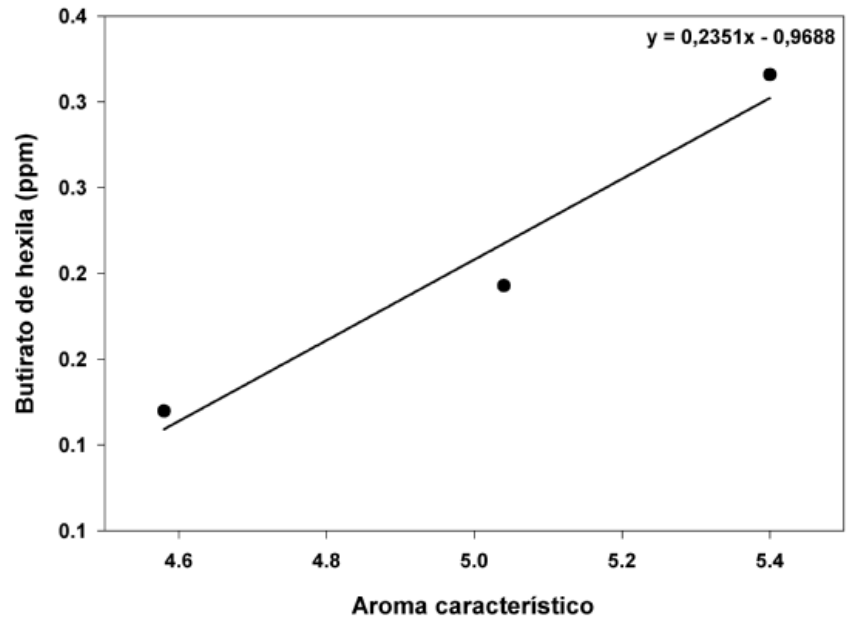

FIGURA 6. Correlação obtida durante o armazenamento, entre o aroma característico e o teor de butirato de hexila.

Para o sabor característico, as correlações foram de 0,34 ( $\alpha=5 \%), 0,68(\alpha=0,1 \%), 0,42(\alpha=0,1 \%)$ e 0,49 $(\alpha=0,1 \%)$, para o butirato de etila, butirato de hexila e caproato de hexila, respectivamente, o que demonstra a importância destes compostos no sabor e aroma do suco de maracujá.

O aroma doce apresentou correlações significativas $(\alpha=0,1 \%)$ com o butirato de etila $(0,52)$, caproato de etila $(0,42$,$) , butirato de hexila (0,40)$ e caproato de hexila $(0,35)$. Conforme FURIA [6], o butirato de etila e o butirato de hexila estão associados às notas doces e frutais. NARAIN e BORA [14] atribuem ao butirato de etila o aro- 
ma doce do suco de maracujá além de denotar um aroma de fruta fresca (aroma característico).

$\mathrm{O}$ aroma floral apresentou correlações significativas $(\alpha=0,1 \%)$ com o butirato de etila $(0,47)$, caproato de etila $(0,50)[11]$, butirato de hexila $(0,54)$ e caproato de hexila $(0,38)$. Segundo PARLIMENT [15], o butirato de etila e o caproato de etila estão associados também ao aroma floral do suco de maracujá.

O aumento da concentração de furfural em suco de frutas têm sido fortemente correlacionado com a degradação do seu flavor característico [4, 10] e à formação de pigmentos escuros [3, 8], principalmente em suco de laranja. De acordo com NAGY \& RANDALL [13], o furfural não é diretamente responsável pela formação de flavors indesejáveis, entretanto, este composto pode ser utilizado como um indice de verificação da qualidade sensorial de sucos, uma vez que uma série de compostos é originada quando da sua formação, durante a degradação do ácido ascórbico. De acordo com SOLOMON, SVANBERG \& SAHLSTRÖM [23] o furfural sofre polimerização como um aldeído ativo e pode combinar-se com aminoácidos, contribuindo para o escurecimento do suco. Em relação ao flavor, BLAIR [2] atribui o seu envolvimento com a formação de compostos indesejáveis, dada a sua combinação com substâncias sulfuradas, as quais estão presentes no maracujá em grandes quantidades [26], levando à formação do tiofurfural, um composto altamente odorífero. Entretanto, a degradação do ácido ascórbico à furfural envolve um série de reações, e grande parte dos compostos intermediários formados, permanece desconhecida [21].

No suco de maracujá-amarelo pasteurizado, e armazenado à temperatura ambiente por quatro meses, FANG, CHEN \& CHIOU [5] observaram um pequeno aumento do conteúdo de furfural, o que foi acompanhado pela degradação da sua qualidade sensorial, fato também constatado por KAANANE, KANE \& LABUZA [8] em suco de laranja.

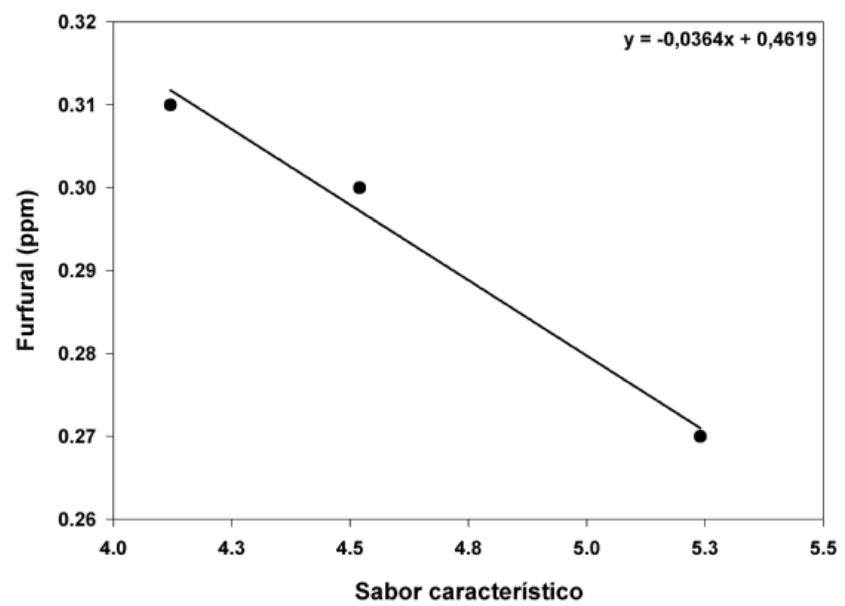

FIGURA 7. Correlação obtida durante o armazenamento, entre o sabor característico e o teor de furfural.

Neste trabalho, indices significativos foram observados entre o furfural e o sabor oxidado $(0,51, \alpha=0,1 \%)$, sabor cozido $(0,43, \alpha=0,1 \%)$, sabor estranho $(0,38$, $\alpha=0,1 \%)$ e o gosto amargo $(0,32, \alpha=5 \%)$, além de correlação negativa com o aroma característico e sabor característico (Figura 7), demonstrando que o aumento da concentração de furfural concomitante à diminuição da intensidade das caracteristicas desejáveis, e ao aumento daquelas consideradas prejudiciais a qualidade sensorial do suco.

\section{4 - CONCLUSÕES}

- Pela análise das correlações, observou-se que os compostos voláteis butirato de etila, butirato de hexila, caproato de etila e caproato de hexila, também a sacarose e a manutenção da cor foram importantes para a preservação da qualidade sensorial do suco.

- A avaliação de alterações sensoriais como o escurecimento utilizando-se métodos instrumentais de avaliação de cor, pode ser efetuada. A variação da cor, indicada pelo valor "a" de Hunter, parece ser o melhor indicador, dadas as correlações significativas observadas entre este parâmetro e as características sensoriais. Entretanto, as correlações obtidas devem ser observadas com cautela, levando sempre em consideração a variabilidade dos dados observados, sem o que, valores de correlação, mesmo que elevados, podem não ser representativos, especialmente quando a análise é conduzida utilizando-se os valores médios experimentais, em detrimento aos valores individuais das repetições.

\section{5 - REFERÊNCIAS BIBLIOGRÁFICAS}

[1] ALMEIDA, T. C. A., FOLEGATTI, M. I. S., FREIRE, M. T. A., MADEIRA, M. S., SILVA, F. T., SILVA, M. A. A. P. Determinação do perfil sensorial e parâmetros de qualidade de figos em calda produzidos pela indústria brasileira. Ciênc. Tecnol. Alimentos, v. 19, n. 2, p. 234-240. 1999.

[2] BLAIR, J. S. Thiofurfural as an important factor in the development of off-flavor in canned orange juice during storage. Citrus Station Mimeo Rpt. CES 65-4. Lake Alfred. Flórida. 1964.

[3] DINSMORE, H. L., NAGY, S. Colorimetric furfural measurement as an index of deterioration in stored citrus juices. Journal of Food Science, v. 37, p. 768-770, 1972.

[4] DINSMORE, H. L., NAGY, S. Fruits and fruit products improved colorimetric determination for furfural in citrus juices. Journal of Association of Official Analitical Chemists, v. 57, p. 332-334. 1974.

[5] FANG, T., CHEN, H-E., CHIOU, L. M. J. Effects of heat treatment and subsequent storage on the quality of passion fruit (Passiflora edulis) juice. Symposium of International Federation of Fruit Juice Producers. Proceedings... Den Haag: [s.n. ], 1986. p. 105-123.

[6] FURIA, T. E. 1975. Handbook of food additives. 2. ed. Cleveland, Ohio, CRC Press.

[7] HUET, R. L'arome du jus de grenadille. Fruits, v. 28, n. 5, p. 397-403, 1973.

[8] KaAnane, A., KAne, D. \& LABUZA, T.P. Time and temperature effect on stability of Morroccan processed 
orange juice during storage. Journal of Food Science, Chicago, v. 53, n. 5, p. 1470-1473, 1988.

[9] MACFIE, H. J. H., HEDDERLEY, D. Current practice in relating sensory perception to instrumental measurements. Food Quality and Preferences, v. 4, p. 41-49, 1993.

[10] MARCY, J. E., ROUSSEF, R. L., High-performance liquid chromatographic determination of furfural in orange juice. Journal of Agricultural and Food Chemistry, v. 32, p. 979-982. 1984.

[11] MORTON, I.D., MACLEOD, A. J. 1990. Food Flavours Part B. The Flavour of Fruits. Developments in Food Science 3C. Ed. Elsevier.

[12] MURRAY, K. E., SHIPTON, J., WHITFIELD, F. B. The chemistry of food flavour. Australian Journal Chemical, v. 25, n. 1, p. 1921-1933, 1972.

[13] NAGY, S., RANDALL, V. Use of furfural content as an index of storage temperature abuse in commercially processed orange juice. Journal of Agricultural and Food Chemistry, v. 21, n. 2, p. 272-275, 1973.

[14] NARAIN, N. , BORA, P. S. Post-harvest changes in some volatile flavour constituints of yellow passion fruit (Passiflora edulis $\mathrm{f}$. flavicarpa). Journal of Science of Food and Agricultural, v. 60, n. 4, p. 529-530, 1992.

[15] PARLIMENT, T. H. Some volatiles constituents of passion fruit. Journal of Agricultural and Food Chemistry, v. 20, n. 5, p. 1043-1045, 1972.

[16] PEDRÃO, M. R., BElEiA, A., MOdesta, R. C. D., PRUDENCIO-FERREIRA, S. H. Estabilidade físico-química e sensorial do suco de limão Tahiti natural e adoçado, congelado. Ciênc. Tecnol. Aliment., v. 19, n. 2, p. 282-286. 1999.

[17] PRIMO, E. Química Agrícola, Alimentos, tomo III. Ed. Alambra, Madrid. 1979.

[18] REMACHA, J. E., IBARZ. A., GINER, J. Evolución del color, por efecto de la temperatura, en pulpas de fruta. Revista Alimentaria, v. n. 4, p. 59-68, 1992.
[19] SANDI, D. Efeito do tratamento térmico e do armazenamento sobre a qualidade sensorial do suco de maracujá-amarelo (Passiflora edulis var. flavicarpa). Viçosa, 1999. 153 p. Dissertação de Mestrado (Mestre em Ciência e Tecnologia de Alimentos) - Departamento de Tecnologia de Alimentos, Universidade Federal de Viçosa (UFV).

[20] SAS. User's guide: basic and statistic. Institute Inc., N. C., USA, 1995.

[21] SAWAMURA, M., NAKAGAWA, S., KATSUNO, S., HAMAGUCHI, H., UKEDA, $H$. The effects of antioxidants on browning and on degradation products caused by dehydroascorbic acid. Journal of Food Science, v. 65, n. 1 , p. 20-23. 2000.

[22] SEAGRI - Secretaria da Agricultura, Irrigação e Reforma Agrária. Bahia. Disponível em <http://www. seagri.ba. gov.br/seagri/Mapa_agricola.asp?qact $=$ prd\&sprdid $=30>$. Consultado em janeiro de 2001.

[23] SOLOMON, O., SVANBERG, U., SAHLSTRÖM, A. Effect of oxygen and fluorescent light on the quality of orange juice during storage at $8^{\circ} \mathrm{C}$. Food Chemistry, v. 53 , p. 363-368. 1995.

[24] STONE, H., SIDEL, J., OLIVER, S., WOOLSEY, A., SINGLENTON, R. C. Sensory evaluation by quantitative descriptive analysis. Food Technology, v. 28, n. 1, p. 24-34, 1974.

[25] TCHANGO TCHANGO, J., TAILIEZ, R., Eb, P. , NJINE, T., HORNEZ, J. P. Heat resistance of the spoilage yeasts Candida pelliculosa and Kloeckera apis and pasteurization values for some tropical fruit juices and nectars. Food Microbiology, v. 14, n. 1, p. 93-99, 1997.

[26] WEBER, B., MOSANDL, A. Stereoisomeric flavour compounds LXXV: synthesis and structure-function relationship of 3-methylthilbutanal enantiomers. Zeitschrift fur Lebensmmittel-Unterssuchung und Forschung, v. 204, p. 194-197, 1997.

[27] YANG, X., PEPPARD, T. Solid-phase microextraction for flavor analysis. Journal of Agricultural and Food Chemistry, v. 42, n. 2, p. 1925-1930, 1994. 